\title{
A New Class of Knots with Property $P$
}

\author{
By \\ Yoko NAKAGAWA*
}

\section{$\S 1$. Introduction}

It is known that several classes of knots have property $P([1],[3],[5]$, [9] ).

In this paper, it will be shown that the special class of knots has property $P$.

To show the above, 3 -dimensional homology spheres constructed by Dehn's method will be considered and it will be shown that they are not 3-dimensional homotopy spheres.

I am grateful to Prof. R. H. Fox and the referee for helpful comments and suggestions regarding this work.

A singular disk in the 3 -sphere $S^{3}$ means a map $f$ of an oriented disk $\bar{D}$ into $S^{3}$. For brevity, one may refer to the image $D=f(\bar{D})$ as the singular disk.

Among the singularities that a singular disk may have perhaps the simplest is a clasping singularity or just clasp. This consists of two mutually disjoint slits $\bar{S}$ and $\overline{\bar{S}}$ that are mapped by $f$ topologically onto an arc $S$ of $D$. The singular disks to be considered are those that have only simple clasps. Let us call such a disk an elementary disk. This is a natural class to consider, since it is known that any singular disk in general position can be deformed, without moving the boundary, into an elementary disk [10].

If $D$ is an elementary disk then a regular neighborhood $W$ of $D$ in $S^{3}$ is a handlebody, and its boundary $\partial W$ is an orientable surface of some

Communicated by N. Shimada, February 12, $1974 . \quad$ Revised April, 1, 1974.

* Graduate School, Kobe University, Kobe. 
genus $g$, where $g$ is the same as the number of clasps. Let us call $D$ totally knotted if $\partial W$ is incompressible* in $S^{3}-\partial D$.

Let $V$ be a tame solid torus in $S^{\mathbf{3}}$, and $a$ a simple closed curve contained in $\partial V$ and not contractible on $\partial V$. Let $\varphi$ be a homeomorphism of a torus $S^{1} \times \partial D^{2}$ onto the torus $\partial V$ which maps $1 \times \partial D^{2}, 1 \in S^{1}$, onto $a$. By this homeomorphism $\varphi$ we will get a new 3-dimensional manifold $M \cong\left(S^{3}-\right.$ int $\left.V\right) \bigcup_{\varphi} S^{1} \times D^{2}$, identifying $x\left(\in S^{1} \times \partial D^{2}\right)$ with $\varphi(x)$.

Let us give a canonical orientation to $S^{3}$ and $M$ in such a way that $M$ and $S^{3}$ induce the same orientation in $S^{3}-\operatorname{int} V$.

Let $m$ and $l$ be a meridian and a longitude of $\partial V$ respectively. We also denote by $m$ and $l$ the elements of $\pi_{1}(\partial V)$ or $\pi_{1}\left(S^{3}-\operatorname{int} V\right)$ represented by these curves. Let $\alpha$ be a curve on $\partial V$ which, when properly oriented, represents the element $m^{\tau} l^{\nu}(\tau, \gamma$ : integers). Since the manifold $M$ will be the homology sphere, $\tau$ must be +1 or -1 . We may assume, changing the orientation of $\alpha$ if necessary, that $\alpha$ represents the element $m l^{\nu}(\gamma \neq 0)$.

In this paper, let us choose only $\alpha$ that is not a meridian, i.e. $a$ does not represent the element $m$ of $\pi_{1}(\partial V)$.

Then the following main result will be proved.

Theorem I. The knot type $k$ which is equivalent to the boundary of a totally knotted disk with two clasping" singularities has property $P$.

Proving this theorem, it is equivalent to prove the following one.

Let $M$ be a homology sphere constructed by the method in the above.

Theorem II. A homology sphere $M$ is not simply connected if the knot type $k$ of the core of $V$ is equivalent to the boundary of a totally knotted disk with two clasping singularities.

\section{Lemma}

A normalized Alexander polynomial means the Alexander polynomial [2] with the smallest positive but no negative powers of each

* $\partial W$ is incompressible in $S^{3}-\partial D$ means the induced map of natural inclusion of $\pi_{1}(\partial I V)$ into $\pi_{1}\left(S^{3}-\partial D\right)$ is a monomorphism. 
generator.

By multiplying or diving by some powers of generators, any Alexander polynomial can be changed to a normalized Alexander polynomial.

To prove theorem II, we need the following lemmas.

Lemma 1. I.et $G$ be a finitely presented group which is isomorphic to the non-trivial free product $G_{1} * G_{2}$, and the abelianized groups of $G_{1}$ and $G_{2}$ be both infinite cyclic groups. If the i-th normalized Alexander polynomial of $G$ is not zero, then it is a product of two one variable polynomials.

Proof. Let an $m_{1} \times n_{1}$-matrix $A_{1}$ and an $m_{2} \times n_{2}$-matrix $A_{2}$ be Alexander matrices of $G_{1}$ and $G_{2}$, respectively. Then an Alexander matrix $A$ of the free product $G$ of $G_{1}$ and $G_{2}$ is the $\left(m_{1}+m_{2}\right) \times\left(n_{1}+n_{2}\right)$ matrix $\left(\begin{array}{cc}A_{1} & 0 \\ 0 & A_{2}\end{array}\right)$. Let $g_{1}$ and $g_{2}$ be generators of the Abelianized group $Z$ of $G_{1}$ and $G_{2}$, respectively. For any integer $t$, the $t$-th polynomials are defined for each $G_{1}$ and $G_{2}$. They are one variable polynomials including the case that the polynomial, is " 1 " or " 0 " after normalization, i. e. the $t$-th polynomial of $G_{1}$ is $\Delta^{(t)}\left(g_{1}\right)=\sum_{i \geqq 0} a_{i} g_{1}^{i}$, and the $t$-th polynomial of $G_{2}$ is $\Delta^{(t)}\left(g_{2}\right)=\sum_{j \geq 0} b_{j} g_{2}^{j}$.

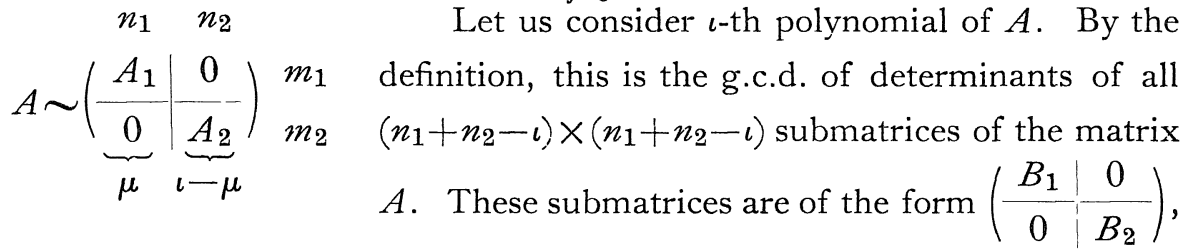
wehre $B_{1}$ is an $\left(n_{1}-\mu\right) \times\left(n_{1}-\mu\right)$ submatrix of $A_{1}$, and $B_{2}$ is an $\left(n_{2}-\imath+\mu\right)$ $\times\left(n_{2}-\imath+\mu\right)$ submatrix of $A_{2}$. The determinant of this form is the product of det $B_{1}$ and det $B_{2}$. Fixing the number $\iota$ and $\mu$, consider all the $\left(n_{1}-\mu\right) \times\left(n_{1}-\mu\right)$ submatrices of $A_{1}$, and the $\left(n_{2}-\iota+\mu\right) \times\left(n_{2}-\imath+\mu\right)$ submatrices of $A_{2}$. Let $p\left(g_{1}\right)$ be the g.c.d. of determinants of the above $\left(n_{1}-\mu\right) \times\left(n_{1}-\mu\right)$ submatrices of $A_{1}$. By the definition, $p\left(g_{1}\right)$ is the $\mu$-th polynomial of $A_{1}$, and $q\left(g_{2}\right)$, similarly defined, is the $(\iota-\mu)$-th polynomial of $A_{2}$. The product $p\left(g_{1}\right) \cdot q\left(g_{2}\right)$ is the g.c.d. of all the determinants of the form $\left(\operatorname{det} B_{1}\right) \cdot\left(\operatorname{det} B_{2}\right)$, since no determinant of any submatrix of $A_{1}$ can 
have any factor in common with the determinant of any submatrix of $A_{2}$. Since $\mu$ ranges over $0,1, \ldots, \iota$, the $\iota$-th polynomial of $A$ is the g.c.d. of $\Delta^{(0)}\left(g_{1}\right) \times \Delta^{(\ell)}\left(g_{2}\right), \quad \Delta^{(1)}\left(g_{1}\right) \cdot \Delta^{(\ell-1)}\left(g_{2}\right), \ldots, \Delta^{(\mu)}\left(g_{1}\right) \cdot \Delta^{(\ell-\mu)}\left(g_{2}\right), \ldots, \Delta^{(\ell)}\left(g_{1}\right)$ $\times \Delta^{(0)}\left(g_{2}\right)$. In general $\Delta^{(t)}$ is divisible by $\Delta^{(t+1)}$. So $\Delta^{(0)}, \ldots, \Delta^{(\ell-1)}$ are divisible by $\Delta^{(\iota)}$. Since $g_{1}$ and $g_{2}$ are different variables, $\Delta^{(\mu)}\left(g_{1}\right)$ and $\Delta^{\left(\mu^{\prime}\right)}\left(g_{2}\right)$ can have no common factor other than a constant, and since $G_{1}$ and $G_{2}$ are abelianized to infinite cyclic groups, neither $\Delta^{(\mu)}\left(g_{1}\right)$ nor $\Delta^{\left(\mu^{\prime}\right)}\left(g_{2}\right)$ can have any constant factor other than 1 . Thus the g.c.d. of the above products, i.e. the $\iota$-th polynomial of $A$, is just the product $\Delta^{\left(\imath-\iota^{\prime \prime}\right)}\left(g_{1}\right) \cdot \Delta^{\left(\imath-\iota^{\prime}\right)}\left(g_{2}\right)$, where $\iota^{\prime}$ and $\iota^{\prime \prime}$ are the smallest number among that the $\left(\iota-\iota^{\prime \prime}\right)$-th and $\left(\iota-\iota^{\prime}\right)$-th Alexander polynomials of $G_{1}$ and $G_{2}$ are non-zero.

Corollary to Lemma 1. Under the same conditions in Lemma 1, the -th normalized Alexander polynomial of $G$ has non-zero constant term.

Proof. It is almost trivial.

Lemma 2. Let $X$ be an orientable connected 3-manifold with bounda$r y$ and the boundary $\partial X$ is a closed surface with genus 2 . If the homomorphism of $\pi_{1}(\partial X)$ into $\pi_{1}(X)$ induced by the natural inclusion map is not a monomorphism, then $\pi_{1}(X)$ is the free product of two non-trivial groups.

Proof. Since the mapping of $\pi_{1}(\partial X)$ into $\pi_{1}(X)$ is not a monomorphism, there exists a disk $D$ is $X$ whose boundary $J$ is a nontrivial closed curve on $\partial X$. By the Loop Theorem [11] and Dehn's Lemma [7], we can assume that $D$ is nonsingular and $J$ is a simple closed curve.

Let us assume first that $J$ is homologous to zero on $\partial X$, i.e. that $J$ is separates $\partial X$ into two surfaces $T_{1}$ and $T_{2}$ with common boundary $J ; T_{1}$ and $T_{2}$ are both surfaces of genus 1 .

Let $D_{1}$ and $D_{2}$ be copies of $D$ in $X$ that have $J$ as common boundary. Along $J$ sew $D_{1}$ to $T_{1}$, and $D_{2}$ to $T_{2}$. Then we get two tori. Let $M_{i}$ be the manifold in $X$ bounded by $D_{i} \cup T_{i}$, thus $M_{i}$ is a 3 -dimensional manifold whose boundary is a torus in $X(i=1,2)$. Since the intersection of $M_{1}$ and $M_{2}$ consists of a nonsingular disk $D$, by the Van Kampen Theorem, $\pi_{1}(X)$ is isomorphic to the nontrivial free product $\pi_{1}\left(M_{1}\right) * \pi_{1}\left(M_{2}\right)$. 
To complete the proof, it will be shown that $J$ must be homologous to zero on $\partial X$. Suppose, to the contrary, that $/$, suitably oriented, represents an element $\alpha$ of $\pi_{1}(\partial X)$ that does not lie in the commutator subgroup $\left[\pi_{1}(\partial X), \pi_{1}(\partial X)\right]$. Then there exists a simple closed curve $J^{\prime}$ on $\partial X$ that intersects $J$ at just one point. Let $\beta$ denote the element of $\pi_{1}(\partial X)$ represented by $J^{\prime}$ (suitably oriented).

Since $\alpha \neq 1$ and $\beta \neq 1$ it follows from a result of Greendlinger [4] that either $\alpha \beta \bar{\alpha} \bar{\beta} \neq 1$ or $\alpha=\gamma^{m}$ and $\beta=\gamma^{n}$ for some element $\gamma$ of $\pi_{1}(\partial X)$ and integers $m$ and $n$.

If, in fact, $\alpha=\gamma^{m}, \beta=\gamma^{n}$ then, since the intersection number $S(\alpha, \beta)=$ $S\left(J, J^{\prime}\right)$ is equal to \pm 1 , and $S(\alpha, \beta)=S\left(\gamma^{m}, \gamma^{n}\right)=m n S(\gamma, \gamma)$, it must be that $m= \pm 1$ and $n= \pm 1$. But this means that $\alpha=\beta^{ \pm 1}$, and hence that $J^{\prime}$ can be deformed into $J$ in the complement of $J$. This contradiction shows that $\alpha \beta \bar{\alpha} \bar{\beta} \neq 1$.

Consequently if $N$ is a regular neighborhood of $J \cup J^{\prime}$ on $\partial X$, then its boundary $\partial N$ is not contractible on $\partial X$. Hence $\partial N$ separates $\partial X$ into two surfaces $N$ and $\partial X-N$, each of genus 1 . As shown in the first part of this proof it follows that $\pi_{1}(X)$ must be the free product of two nontrivisl groups.

Lemma 3. Let $X$ be an orientable connected 3-manifold with boundary, $i: \partial X \rightarrow X$ an inclusion map and $i_{*}: H_{1}(\partial X) \rightarrow H_{1}(X)$ an induced map. Then the rank of the kernel of $i_{*}$ is exactly half of the rank of $H_{1}(\partial X)$.

Proof. Let us consider the following exact sequences:

$$
\begin{aligned}
0 & \longrightarrow H_{3}(X, \partial X) \rightarrow H_{2}(\partial X) \rightarrow H_{2}(X) \\
& \rightarrow H_{2}(X, \partial X) \rightarrow H_{1}(\partial X) \stackrel{i *}{\longrightarrow} H_{1}(X) \\
& \longrightarrow H_{1}(X, \partial X) \longrightarrow H_{0}(\partial X) \rightarrow H_{0}(X) \rightarrow 0
\end{aligned}
$$

Let $2 g$ be the rank of $H_{1}(\partial X), a$ of $H_{0}(\partial X), b$ of $H_{1}(X, \partial X)$ and $c$ of $H_{1}(X)$.

By Poincaré Duality $H_{2}(\partial X)$ has rank $a, H_{2}(X)$ has rank $b$ and $H_{2}(X, \partial X)$ has rank $c$. Also $H_{3}(X, \partial X)$ has the same rank 1 as $H_{0}(X)$. Among the numbers $a, b, c, 1$ and $g$, by the exactness, there is an equation 
$2 g=(c-b+a-1)+(c-b+a-1)$. Moreover from the exactness, the rank of ker $i_{*}$ is $c-b+a-1$; this is just half of the rank of $H_{1}(\partial X)$, i.e. $g=c-b+a-1$.

\section{$\S 3$. Proof of the Theorem}

Proof of the TheoremII. Let $k$ be a knot which is equivalent to the boundary of a totally knotted disk $E$ with two clasping singularities, $V$ a regular neighborhood of $k$ and $W$ a regular neighborhood of $E$ in $S^{3}$. The homology sphere $\left(S^{3}\right.$-int $\left.V\right) \cup_{\varphi} S^{1} \times D^{2} \cong\left(S^{3}\right.$-int $\left.W\right) \cup\left((W\right.$-int $V) \cup_{\varphi}$ $\left.S^{1} \times D^{2}\right)$ will be denoted by $M$, where $\varphi$ is a homeomorphism of $S^{1} \times \partial D^{2}$ onto $\partial V$ which maps $1 \times \partial D^{2}, 1 \in S^{1}$, onto $\alpha$, where $\alpha$ is a simple closed curve on $\partial V$ and not a meridian curve of $\partial V$.

Since $\partial W$ is incompressible in $S^{3}-k$ and $S^{3}-\operatorname{int} W \subset S^{3}-k$, the map from $\pi_{1}(\partial W)$ into $G_{1}=\pi_{1}\left(S^{3}-\operatorname{int} W\right)$ is a monomorphism. To show that $\pi_{1}(M)$ is not trivial, by the Van Kampen Theorem, it is enough to show that a map from $\pi_{1}(\partial W) \cong \pi_{1}\left(\partial\left((W-\operatorname{int} V) \cup S^{1} \times D^{2}\right)\right)$ into $G_{2}=\pi_{1}((W-$ $\left.\operatorname{int} V) \cup S^{1} \times D^{2}\right)$ is a monomorphism. If both maps from $\pi_{1}(\partial W)$ into $G_{1}$ and $G_{2}$ are monomorphisms, then $\pi_{1}(M)$ is isomorphic to the free product with amalgamation $G_{1_{\pi_{1}}(\tilde{\partial} W)} G_{2}$.

Let us consider $G_{2}=\pi_{1}\left((W-\operatorname{int} V) \cup S_{\varphi}^{1} \times D^{2}\right)$. Let $W$ be a handlebody with two handles, which may be knotted or linked in $S^{3}$. To calculate $G_{2}$, it is enough to consider a handlebody $W^{\prime}$ in standard position in $S^{3}$; thus there is an autohomeomorphism of $S^{3}$ which maps $W$ onto $W^{\prime}$, whose two handles are neither knotted nor linked with each other. By this mapping, $V$ is mapped onto $V^{\prime} \subset W^{\prime}$ and $\alpha$ is mapped onto $\alpha^{\prime} \subset \partial V^{\prime}$. The simple closed curve $a^{\prime}$ is not a meridian of $\partial V^{\prime}$.

Let us construct another homeomorphism of $W^{\prime}$ onto a handlebody $W^{\prime \prime}$ as follows: take two meridian cells in $W^{\prime}, m_{1}$ and $m_{2}$. Cut $W^{\prime}$ along these meridian cells and turn the exposed faces a suitable number of times, to untwist $V^{\prime}$, and sew back together again. Then we get a new solid torus $V^{\prime \prime}$ in $W^{\prime \prime}$. By this homeomorphism, $\alpha^{\prime}$ is mapped onto $\alpha^{\prime \prime}$ on $\partial V^{\prime \prime}$. The curve $\alpha^{\prime \prime}$ is not a meridian curve of $\partial V^{\prime \prime}$. With the appropriate orientation $\alpha^{\prime \prime}$ represents an element of $\pi_{1}\left(\partial V^{\prime \prime}\right)$ of the form $m l^{\nu}$, where $m$ 


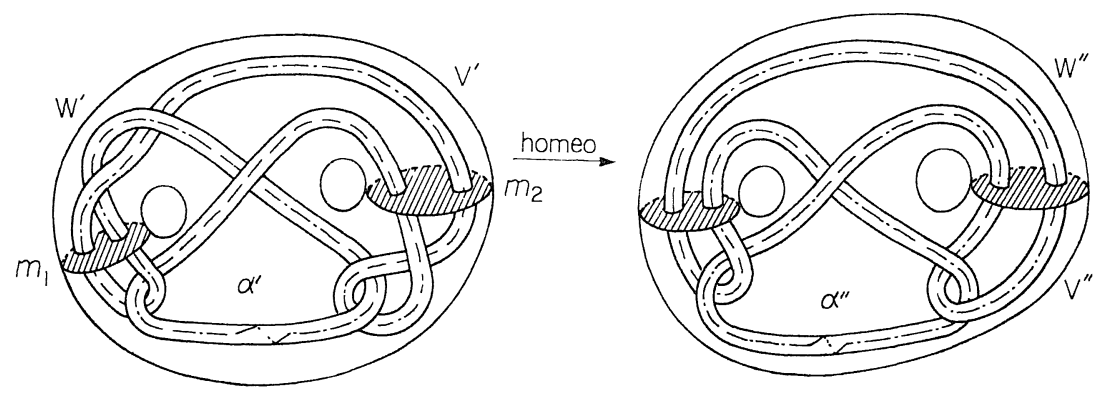

Fig. 1

is represented by a meridian, and $l$ by a longitude of $\partial V^{\prime \prime}$. Since $\alpha^{\prime \prime}$ is not a meridian, $\nu$ is a non-zero integer. Then the manifold $\left(W^{\prime}-\operatorname{int} V^{\prime}\right)$ $\bigcup_{\varphi^{\prime}} S^{1} \times D^{2}$ is homeomorphic to the manifold $\left(W^{\prime \prime}-\operatorname{int} V^{\prime \prime}\right) \underset{\varphi^{\prime \prime}}{\cup} S^{1} \times D^{2}$, $\varphi^{\prime}$ where $\varphi^{\prime \prime}$ is a homeomorphism of $S^{1} \times \partial D^{2}$ onto $\partial V^{\prime \prime}$ that maps $1 \times \partial D^{2}$, $l \in S^{1}$, onto $\alpha^{\prime \prime}$.

Thus the manifold ( $W$-int $V$ ) $\cup S^{1} \times D^{2}$ is seen to be homeomorphic, by the composition of above maps, to the manifold ( $\left.W^{\prime \prime}-\operatorname{int} V^{\prime \prime}\right) \cup S^{1} \times D^{2}$.

Let us assume that the map from $\pi_{1}(\partial W)$ into $\pi_{1}\left((W-\operatorname{int} V) \cup^{\varphi^{\prime \prime}} S^{1} \times D^{2}\right)$ is not a monomorphism. Since $(W$-int $V) \cup S^{1} \times D^{2}$ is homeomorphic to $\left(W^{\prime \prime}-\operatorname{int} V^{\prime \prime}\right) \cup S^{1} \times D^{2}$ it is equivalent to assume that the map of $\left.\pi_{1}\left(\partial\left(W^{\prime \prime}-\operatorname{int} V^{\prime \prime}\right) \bigcup_{\varphi^{\prime \prime}}^{\varphi^{\prime \prime}} S^{1} \times D^{2}\right)\right) \cong \pi_{1}\left(\partial W^{\prime \prime}\right)$ into $\pi_{1}\left(\left(W^{\prime \prime}-\operatorname{int} V^{\prime \prime}\right) \underset{\varphi^{\prime \prime}}{\cup} S^{1} \times D^{2}\right)$ is not a monomorphism.

By lemma 2, there exist manifolds $M_{1}$ and $M_{2}$ such that $M_{1} \cup M_{2}$ $=\left(W^{\prime \prime}-\operatorname{int} V^{\prime \prime}\right) \cup_{\varphi^{\prime \prime}} S^{1} \times D^{2}, \quad M_{1} \cap M_{2}=$ nonsingular disk, and both the boundaries $\partial M_{1}$ and $\partial M_{2}$ are tori; then $\pi_{1}\left(M_{1} \cup M_{2}\right)$ is isomorphic to the nontrivial free product $\pi_{1}\left(M_{1}\right) * \pi_{1}\left(M_{2}\right)$. By lemma 3 , since $M_{i}$ is a 3 -manifold whose boundary is a torus, $H_{1}\left(M_{1}\right)$ and $H_{1}\left(M_{2}\right)$ are not tivial. Since $\pi_{1}\left(M_{1} \cup M_{2}\right) /\left[\pi_{1}\left(M_{1} \cup M_{2}\right), \pi_{1}\left(M_{1} \cup M_{2}\right)\right]$ is $Z \times Z$, both $H_{1}\left(M_{1}\right)$ and $H_{1}\left(M_{2}\right)$ are infinite cyclic groups. So by Cor. to lemma 1 , the $\iota$-th polynomial of the free product $\pi_{1}\left(M_{1}\right) * \pi_{1}\left(M_{2}\right)$ must have non-zero constant term.

Let us calculate the polynomial of the group $\pi_{1}\left(\left(W^{\prime \prime}-\operatorname{int} V^{\prime \prime}\right) \bigcup_{\varphi^{\prime \prime}}\right.$ $\left.S^{1} \times D^{2}\right)$. To complete the proof it is enough to consider four different cases; that are depending on the order of the inverse images of slits along knot and the intersection number $S(k, E)$ at the end of slit. 


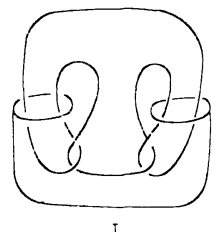

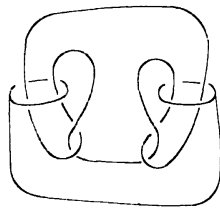

II

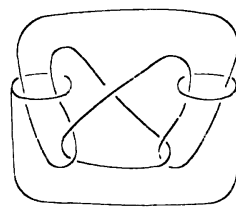

III

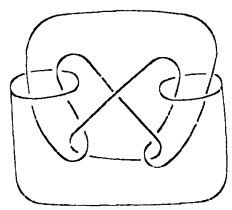

IV

Fig. 2

Take the first case.

To consider the group $\pi_{1}\left(\left(W^{\prime \prime}-\operatorname{int} V^{\prime \prime}\right) \cup \bigcup_{\varphi^{\prime \prime}} S^{1} \times D^{2}\right)$ for this case is equivalent considering the fundamental group of the complement of the graph in Fig. 2 with more relations corresponding to a homeomorphism $\varphi^{\prime \prime}$ of $S^{1} \times \partial D^{2}$ onto $\partial V^{\prime \prime}$ that maps $1 \times \partial D^{2}, l \in S^{1}$, onto $\alpha^{\prime \prime}$ representing an element of $\pi_{1}\left(\partial V^{\prime \prime}\right)$ of the form $m l^{\nu}(\nu \neq 0)$, where $m$ is represented by a meridian, and $l$ by a longitude of $\partial V^{\prime \prime}$.

Let $a, x, m, n$ and $s$ be the gener-

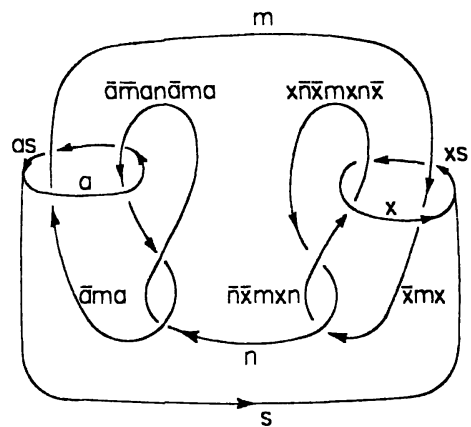

Fig. 3 ators. From Fig. 3 we will get relations: 1) $\bar{m} a s m=\bar{a} \bar{m} a \bar{n} \bar{a} m a \bar{m} a n \bar{a} m a$

2) $\bar{a} \bar{m} a n \bar{a} m a=\bar{m} a n \bar{a} m a \bar{n} \bar{a} m$

3) $\bar{m} \times s m=x \bar{n} \bar{x} \bar{m} \times n \times \bar{n} \bar{x} m \times n \bar{x}$ 4) $x \bar{n} \bar{x} m \times n \bar{x}=\bar{n} \bar{x} m \times n \bar{x} \bar{m} x n$ Let $l$ be a longitude of $\partial V^{\prime \prime}$; then $l$ is denoted by $l=\not h \cdot \not{\mu} \bar{m} a \bar{n} \bar{a} m \not h \cdot \not h \cdot \bar{a} \bar{m} a$ $\times \bar{n} \bar{x} \bar{m} x n \cdot x \cdot \bar{n} \bar{x} m^{4}$.

Corresponding to the map $\varphi^{\prime \prime}$, we need one more relation: $m l^{\nu}=1$, i.e. $\pi_{1}\left(\left(W^{\prime \prime}-\operatorname{int} V^{\prime \prime}\right) \cup \bigcup_{\varphi^{\prime \prime}} S^{1} \times D^{2}\right) \cong\{a, x, m, n, s, l:$

1) $\bar{m} a s m=\bar{a} \bar{m} a \bar{n} \bar{a} m a \bar{m} a n \bar{a} m a$

2) $\bar{a} \bar{m} a n \bar{a} m a=\bar{m} a n \bar{a} m a \bar{n} \bar{a} m$

3) $\bar{m} \times s m=x \bar{n} \bar{x} \bar{m} \times n \times \bar{n} \bar{x} m \times n \bar{x}$

4) $x \bar{n} \bar{x} m \times n \bar{x}=\bar{n} \bar{x} m \times n \bar{x} \bar{m} \times n$

5) $m l^{\nu}=1$

6) $\left.l=\bar{m} a \bar{n} \bar{a} m \bar{a} \bar{m} a \bar{n} \bar{x} \bar{m} \times n \times \bar{n} \bar{x} m^{4}\right\}$.

Since abelianized group of $\pi_{1}\left(\left(W^{\prime \prime}-\right.\right.$ int $\left.\left.V^{\prime \prime}\right) \cup \bigcup_{\varphi^{\prime \prime}} S^{1} \times D^{2}\right)$ is $Z \times Z$, whose generators are represented by $a$ and $x$, the each entry of the Alexander 
matrix is a polynomial with two variables at most. By the free calculus, the Alexander matrix $A$ is equivalent to the following one:

$$
\begin{aligned}
A & \sim\left(\begin{array}{cccccc}
0 & 0 & -1+a & 1-a & a & 0 \\
0 & 0 & -1 & 1 & 0 & 0 \\
0 & 0 & 0 & 0 & x & 0 \\
0 & 0 & 1 & -1 & 0 & 0 \\
0 & 0 & 1 & 0 & 0 & \nu \\
0 & 0 & \bar{a}+\bar{x}-4 & a+x & 0 & 1
\end{array}\right) \\
& \sim\left(\begin{array}{ccccc}
0 & 0 & 0 & a & 0 \\
0 & 0 & 0 & x & 0 \\
0 & 0 & 0 & 0 & 0 \\
0 & 0 & 1 & 0 & \nu \\
0 & 0 & \bar{a}+\bar{x}-4+a+x & 0 & 1
\end{array}\right) \\
& \sim\left(\begin{array}{cccc}
0 & 0 & 1 & \nu \\
0 & 0 & \bar{a}+\bar{x}-4+a+x & 1
\end{array}\right) .
\end{aligned}
$$

Then we get the second polynomial,

$$
\Delta^{(2)}(a, x)=(4 \nu+1) a x-\nu(x+a)-\nu\left(a x^{2}+a^{2} x\right) .
$$

For the other three cases, we get similarly:

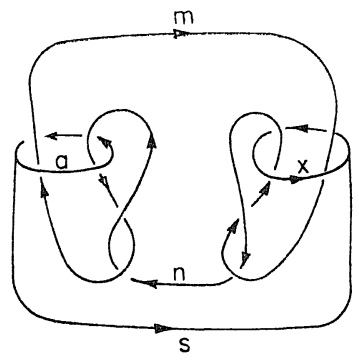

$$
\begin{aligned}
& \pi_{1}\left(\left(W^{\prime \prime}-\operatorname{int} V^{\prime \prime}\right) \cup S^{\prime \prime} \times D^{2}\right)= \\
& \left\{a, x, m, n, s, l:{ }^{\prime \prime}\right.
\end{aligned}
$$

$\bar{m}$ a s $m=\bar{a} \bar{m} a \bar{n} \bar{a} m a \bar{m} a n \bar{a} m a$

$\bar{a} \bar{m} a n \bar{a} m a=\bar{m} a n \bar{a} m a \bar{n} \bar{a} m$

$\bar{m} x s m=\bar{x} \bar{m} x \bar{n} \bar{x} m x \bar{m} \times n \bar{x} m x$

$\bar{x} \bar{m} x n \bar{x} m x=\bar{m} \times n \bar{x} m x \bar{n} \bar{x} m$

$m l^{\nu}=1$

$l=m a \bar{n} \bar{a} \bar{m} \bar{a} \bar{m} a \bar{x} m x \bar{m} \times n \bar{x} m\}$.

$$
A \sim\left(\begin{array}{cccccc}
0 & 0 & -1+a & 1-a & 0 & a \\
0 & 0 & -1 & 1 & 0 & 0 \\
0 & 0 & -1+x & 1-x & 0 & x \\
0 & 0 & -1 & 1 & 0 & 0 \\
0 & 0 & 1 & 0 & \nu & 0 \\
0 & 0 & \bar{a}-\bar{n} & a-x & 1 & 0
\end{array}\right)
$$




$$
\sim\left(\begin{array}{cccc}
0 & 0 & 1 & \nu \\
0 & 0 & \bar{a}-\bar{x}+a-x & 1
\end{array}\right)
$$

Then, $\Delta^{(2)}(a, x)=\nu\left(a x^{2}-a^{2} x\right)+a x+\nu(a-x)$.

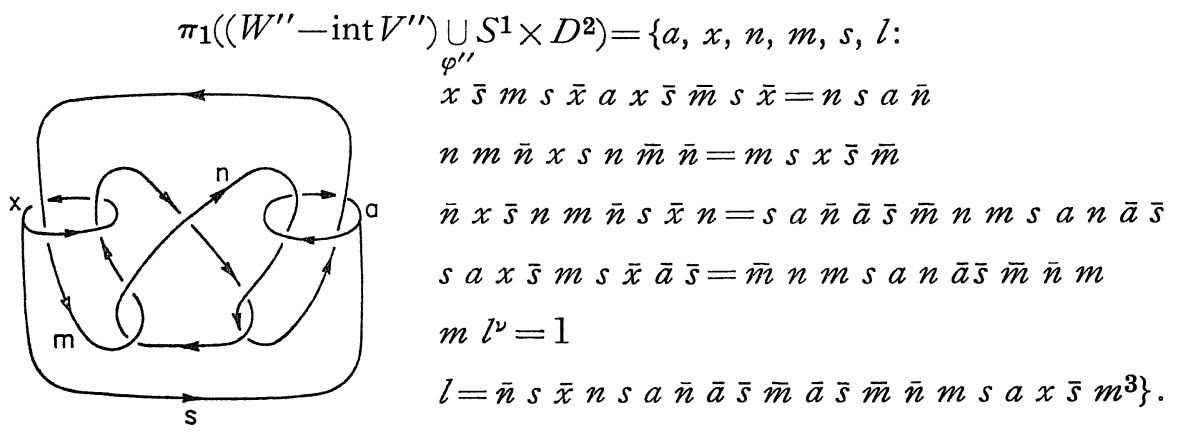

$$
\begin{aligned}
& A \sim\left(\begin{array}{cccccr}
0 & 0 & x-a x & -1+a & 0 & -1 \\
0 & 0 & 0 & 0 & 0 & -1 \\
0 & 0 & x & -1 & 0 & 0 \\
0 & 0 & a x & -a & 0 & 0 \\
0 & 0 & 1 & 0 & \nu & 0 \\
0 & 0 & \bar{x}-3 & \bar{a} \bar{x}+a \bar{x}-\bar{x}+1 & 1 & 0
\end{array}\right) \\
& \sim\left(\begin{array}{cccc}
0 & 0 & 1 & \nu \\
0 & 0 & x-3+\bar{a}+a-1+x & 1
\end{array}\right) .
\end{aligned}
$$

Then $\Delta^{(2)}(a, x)=\nu\left(a^{2} x+a x^{2}\right)+(1-4 \nu) a x+\nu(x+a)$.

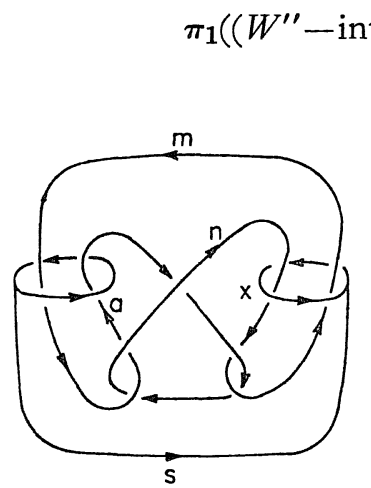

$\left.\left(V^{\prime \prime}\right) \bigcup_{\varphi^{\prime \prime}} S^{1} \times D^{2}\right)=\{a, x, m, n, s, l:$

$m a s \bar{m}=a n \bar{a} m a \bar{n} a n \bar{a} \bar{m} a \bar{n} \bar{a}$

$m \times s \bar{m}=n \times \bar{n}$

$\bar{x} n x=\bar{n} a n \bar{a} m a \bar{n} \bar{a} n \bar{x} m x \bar{n} a$

$\times n \bar{a} \bar{m} a \bar{n} \bar{a} n$

$\bar{a} \bar{m} a n \bar{a} m a=\bar{x} \bar{m} x \bar{n} a n \bar{a} m a \bar{n} \bar{a}$

$\times n \bar{x} m x$

$m l^{\nu}=1$

$l=x \bar{n} a n \bar{a} \bar{m} a \bar{n} \bar{a} n \bar{x} \bar{a} m a \bar{x} \bar{m} x$

$\times \bar{n} a n \bar{a} m\}$. 


$$
\begin{aligned}
A & \sim\left(\begin{array}{cccccc}
0 & 0 & 0 & 0 & 0 & a \\
0 & 0 & 1-x & -1+x & 0 & x \\
0 & 0 & -\bar{x} & \bar{x} & 0 & 0 \\
0 & 0 & -1 & 1 & 0 & 0 \\
0 & 0 & 1 & 0 & \nu & 0 \\
0 & 0 & -\bar{a}+\bar{x}-1+x & 1-a & 1 & 0
\end{array}\right) \\
& \sim\left(\begin{array}{cccc}
0 & 0 & 1 & \nu \\
0 & 0 & -\bar{a}+\bar{x}-a+x & 1
\end{array}\right) .
\end{aligned}
$$

Then $\Delta^{(2)}(a, x)=\nu\left(a^{2} x-a x^{2}\right)+a x+\nu(x-a)$.

In every case, the second normalized polynomial $\Delta^{(2)}$ can not have a constant term.

This is a contradiction, i.e. the first assumption is not true. Then the inclusion map from $\pi_{1}\left(\partial W^{\prime \prime}\right)$ into $\pi_{1}\left(\left(W^{\prime \prime}-\operatorname{int} V^{\prime \prime}\right) \bigcup_{\varphi^{\prime \prime}} S^{1} \times D^{2}\right)$ is a monomorphism.

This completes the proof.

\section{References}

[1] Bing, R. H. and Martin, J. M., Cubes with knotted holes, Trans. Amer. Math. Soc., 155, (1971), 217-237.

[ 21 Crowell, R. H. and Fox, R. H., Introduction to Knot Theory, (Ginn and Company, 1963).

[3] González-Acuña, F., Dehn's construction of knots, Bol. Soc. Mat. Mexicana, 15, (1970), 58-79.

[4] Greendlinger, Problem of conjugacy and coincidence with the anticenter in group theory, Sib. Matem, 2h, 7, (1966).

[ 5 ] Hemple, J., A simply connected 3-manifold is $\mathrm{S}^{3}$ if it is the sum of a solid torus and the complement of a torus knot, Proc. Amer. Math. Soc., 15, (1964), 195-199.

[6] Nakagawa, Y., Elementary Disks and Their Equivalences, Dissertation, Princeton Univ., (1973)

[7] Papakyriakopoulos, C. D., On Dehn's Lemma and the Asphericity of Knots, Ann. of Math., 66, (1957), 1-26.

[8] Seifert, H. and Threlfall, W., Lehrbuch der Topologie, reprint, New York: Chelsea, (1945).

[9] Simon, J., Some classes of knots with property P, Topology of Manifolds, Markham, (1970).

[10] Smythe, N., Handlebodies in 3-Manifolds, Proc. Amer. Math. Soc., 26, (1970).

[11] Stallings, J., On the Loop Theorem, Ann. of Math., 72, (1960), 12-19. 
